\title{
Documents notariés concernant Toussaint
} Desmares

\section{Marie-Françoise Baverel-Croissant}

\section{(2) OpenEdition}

1 Journals

Édition électronique

URL : http://journals.openedition.org/ccibp/606

DOI : $10.4000 /$ ccibp.606

ISSN : 2493-7460

Éditeur

Centre international Blaise Pascal

\section{Édition imprimée}

Date de publication : 27 février 1993

Pagination : 59-68

ISSN : 0249-6674

\section{Référence électronique}

Marie-Françoise Baverel-Croissant, « Documents notariés concernant Toussaint Desmares », Courrier du Centre international Blaise Pascal [En ligne], 15 | 1993, mis en ligne le 07 janvier 2016, consulté le 10 décembre 2020. URL : http://journals.openedition.org/ccibp/606 ; DOI : https://doi.org/10.4000/ccibp. 606

Ce document a été généré automatiquement le 10 décembre 2020.

Centre international Blaise Pascal 


\title{
Documents notariés concernant Toussaint Desmares
}

\author{
Marie-Françoise Baverel-Croissant
}

\section{NOTE DE L'AUTEUR}

Que Madame Régine Pouzet, qui relut patiemment et corrigea nos erreurs de transcription, trouve ici l'expression de nos vifs remerciements.

$1 \mathrm{Au}$ cours de nos recherches dans le Minutier central des notaires parisiens, dont le professeur Jean Mesnard ${ }^{1}$ a montré l'intérêt pour la recherche littéraire, nous avons trouvé cinq actes notariés passés par l'oratoire Toussaint-Joseph-Guy Desmares, prédicateur talentueux, dont la vie, brièvement relatée par Batterel ${ }^{2}$, est beaucoup mieux connue depuis les travaux de Jean Lesaulnier ${ }^{3}$ qui mentionne ces actes, passés à l'étude de Philippe Galloys ${ }^{4}$.

2 Que Toussaint Desmares ait choisi ce notaire n'a rien qui doive surprendre. Cet « ancien notaire distingué par une rare probité et un amour de la justice et de la vérité, qu'il étendoit sur ceux qui souffroient pour l'une et pour l'autre, avoit osé recevoir la protestation de M. Arnauld le Docteur [...]. Et, lorsque M. le Chancellier voulut lui en faire des reproches, il répondit généreusement qu'il n'avoit fait que ce qui est de sa profession, et rien contre les ordres ou le service du Roi ${ }^{5} . .$. ».

\section{Actes $\mathrm{n}^{\circ} 1$}

Le 20 février $1664^{6}$, Toussaint Desmares, par-devant Galloys, notaire et gardenotte $\mathrm{du}$ roi au Châtelet de Paris $^{7}$, cède à Jean Dezaleux, clerc $^{8}$, contre une pension annuelle et viagère de cent livres tournois, la commende de son "prieuré de Saincte Avoye, ses appartenances et deppendances ${ }^{9}$.

1664 vingt febvrier

Par-devant les notaires gardenottes du roy nostre sire en / son chastelet de Paris soubzsignés, furent présents en leurs personnes $\mathrm{M}^{\text {re10 }} /$ Toussainsts Desmares, 
prestre du dioceze de Bayeux, docteur en théologie, prieur / commandataire du Prieuré Saincte-Avoye ${ }^{11}$, anciennement / Nostre Dame de Beaulieu de Saint-Remy, / ordre de Sainct-Benoist, dioceze de Paris, estant de présent à Paris logé / au faux bourg Sainct-Germain, rue de Seyne, à l'hostel de Liancourt, paroisse / SainctSulpice, et M. Jean Dezaleux, clerc dud. dioceze de Paris, / y demeurant, rue du Marturey ${ }^{12}$, parroisse Sainct-Jean-en-Grève, lesquels / volontairement ont faict et constituté leurs procureurs ${ }^{13}$ généraux / spéciaux ${ }^{14}$. /

Ausquels et à chacun d'eux, seul pour le tout, ils ont donné et donnent / pouvoir de pour et es noms desd. sieurs constituants, scavoir / dud. sieur Desmares, résigner entre les mains de Nostre sainct / père le pape, Monseigneur son vice-chancellier ou autre ayant / pouvoir, ledit Prieuré de Saincte Avoye ses appartenances et deppendances et cedder la commande d'iceluy en faveur toutesfois / dud. sieur Dezaleux, et non d'autre, et à la charge d'une / pension annuelle et viagère franche, quitte et exampte de touts / deteors, subciddes ${ }^{15}$ et autres charges quelzconques imposées / ou à imposer, pour quelque cause et occasion que ce soit, / quand bien se seroit par autorité du roy ou ordonnances / de nos seigneurs du Clergé, de la somme de cent livres / tournoiz ${ }^{16}$ que led. sieur Desmares s'est réservée sur condition et / chacuns lesd. fruicts ${ }^{17}$ dud. bénéfice payable par chacun an, / en cette ville de Paris par led. sieur Dezaleux et / ses successeurs, en un seul terme qui sera au jour de Pasques $/ \mathrm{f}^{\circ} 1 \mathrm{v}^{\circ} /$ de chacune année, le premier terme escheu / à pareil jour de Pasques de l'année que l'on comptera seize cent / soixante-cinq, pour, de là en avant, continuer d'an en an à pareil / jour la vie durant dud. sieur Desmares et à la charge ${ }^{18}$ que, / pour plus grande seureté de lad. pension, led. sieur Dezaleux / donnera pour caution la personne de $\mathrm{M}^{\mathrm{r}}$ François Delazeux, / advocat en la cour de Parlement, son frère, qui s'y obligera ${ }^{19}$ avecq / luy solidairement soubz les renonciations requises et en fera / son propre faict et debte ${ }^{20}$, et non autrement, et pour / et au nom dud. sieur Dezaleux, aussy tôt qu'il sera pourveu / dud. Prieuré de Sainct Avoye en conséquence de la présente / désignation, consentir à la création et omologation de lad. / pension de cent livres tournois, franche, quitte, exempte et / payable comme il est dit cy-dessus, et respectueusement / consentir que, sur les présentes résignation et réservation ${ }^{21} /$ de pension, toutes lettres ${ }^{22}$ soient bien et deuement expédiées / par soin ou besoin. Sera jurer et affirmer aud. sr. lesd. sieurs constituants qu'esd. présente n'est intervenu et / n'interviendra aucun dol, fraude, symonie ny autre / paction ${ }^{23}$ ilicite et générallement promettant, s'obligeant etc. Faict / et passé à Paris es étude des notaires l'an mil six-cent / soixante-quatre, le vingtième jour de febvrier avant midy / et ont signé. /

Desmares /J. Dezaleux / Symonnet / Galloys /

Grosse $^{24}$ / Et à l'instant, par-devant lesd. notaires soubzsignés, est comparu / en sa personne $\mathrm{M}^{\mathrm{re}}$ François Dezaleux, advocat en Parlement ${ }^{25}, / \mathrm{f}^{\circ} 2 \mathrm{r}^{\circ} /$ demeurant à Paris, au fauxbourg Sainct-Germain, rue de Seyne, / à l'hostel de Liancourt, parroisse St-Sulpice, lequel s'est / volontairement constitué caution pour led. sr. Jean Dezaleux, / son frère, envers led. sieur Desmares à ce présent et acceptant / des cent livres tournois de pension porté par la résignation / cy-dessus et ci avecq led. sieur son frère a promis solidairement / sans divisions, discussions ny fidejussion ${ }^{26}$, renonçant aux / cent livres tournois de pension par chacun an aud. sieur / Desmares, en ceste ville de Paris, sa vie durant, / aux termes et selon qu'il est porté pour lad. resignation / faisant de lad. pension son propre faict et debte / pour led. sr. Jean Dezaleux, son frère, elizant à ceste / fin son domicile ${ }^{27}$ irrévocable en la maison où / il est comme dû est demeurant auquel lieu etc. nonobstant etc. / s'obligeant etc. Faict et passé à Paris en / estude lesd. jour et an cy-dessus et ont signé. /

Desmares /J. Dezaleux / Symonnet / J. Dezaleux / Galloys /

Par acte passé par-devant Charles et Levasseur Le Jeune, notaire, le premier apvril

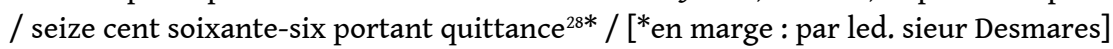
/ appert, led. $\mathrm{M}^{\mathrm{e}}$ Jean Dezaleux et led. sr. F. Dezaleux, / advocat, son frère, estre 
quittes et deschargez de lad. pension de cent livres qui / est estainte et admortye selon et ainsy qu'il est plus au long esnoncé en lad. quittance /

[...] audit. Levasseur dont est apparu [...] / par les notaires soubzsignés ce vingt apvril seize cent soixante-six. /

Symonnet / Galloys /

\section{Acte $\mathrm{n}^{\circ} 2$}

Le deuxième acte, en date du 20 juin $1665^{29}$, témoigne du soin que les Liancourt mettaient à pouvoir aux besoins de leur hôte.

1665 Quictance $^{30}$. Vingt juin. /

Par-devant les notaires gardenottes du roy nostre sire au Chastelet de Paris / soubzsignés, fut présent en sa personne $\mathrm{M}^{\text {re }}$ Toussainct Desmares, prestre, demeurant à / Lyancourt, estant de présent logé en l'hostel de Liancourt, faubourg St-Germain, / parroisse St-Sulpice, lequel a recogneu et confesse avoir receu de très-haut et très- / puissant seigneur Monseigneur Roger Duplessey et seigneur de Liancourt, / duc de La Rocheguyon, et de très-haute et très-puissante Madame Jeanne / de Schomberg, son espouze ${ }^{31}$, par les mains de $\mathrm{M}^{\mathrm{r}}$ Pierre Desnoz, trésorier / de leurs maisons, demeurant en leur hostel, à ce présent, qui luy a baillé, payé, compté,/ nombré et deslivré par lesd. notaires en louis d'argent, le tout bon, / la somme de treize mil huict cent quatre-vingt-deux livres quinze solz, / scavoir dix mil livres pour arreraiges ${ }^{32}$ et pour principal ${ }^{33}$ de cinq cents livres / de rente par lesd. seigneur et dame constituez aud. sieur Desmares par leur promesse / du 22 febvrier seize cent cinquante-six, recogneue le premier apvril aud. an, par / acte estant ensuite passé par-devant Lecaron et Galloys, notaires, moyennant neuf / mil livres à raison du denier dix-huict ${ }^{34}$ suppléés moyennant mil livres / par acte estant ensuite du six apvril 1660, six cent soixante et quatre / livres et cinq sols pour les arreraiges qui en sont deubz et escheuz depuis le $22^{\text {ème }} /$ febvrier seize cent soixante-deux jusques au jourd'huy et estant du passé, et deux mil / deux cent dixhuict livres dix solz contenus en la promesse desd. seigneur et dame de / Liancourt du huict juin 16 soixante-quatre de laquelle susd. somme de / treize mil huict cent quatre-vingt-deux livres quinze solz que led. sr. Desmares / se tient content ${ }^{35}$ et en quitte lesd. seigneur et dame de Liancourt, lesd. sieurs / Desnoz et tous autres, et, ce faisant, il a présentement rendu ausd. sr. Desnoz lesd. / deux promesses comme nulles et acquitées sans prejudice ${ }^{36}$ aud. sr. Desmares / de cent livres de rente qui luy sont encore deubz promettant etc., s'obligeant etc. Faict et / passé à Paris aud. hostel de Liancourt le vingtième jour de juin après midy / l'an mil six cent soixantecinq et ont signé. /

Lecaron / Desmares / Desnoz / Galloys /

\section{Acte $n^{\circ} 3$}

Les constitutions de rente étaient fréquentes au XVII ${ }^{e}$ siècle. Par un acte du 6 juillet $1665^{37}$ , le Père Noël de Lalane ${ }^{38}$ constitue une rente de cinq cent cinquante livres à Toussaint Desmares, le montant de cette rente étant assuré sur les revenus et profits des biens que possédait Noël de Lalane au Mesnil-le-Roi. Au bas de l'acte, sa signature, « Lalane abbé de Valcroissant ", atteste l'importance qu'il attachait à cette abbaye cistercienne située non loin de Die (Drôme). Noël de Lalane était en effet abbé de deux des abbayes cisterciennes : Bonlieu $^{39}$ et Valcroissant. Bien que subsistât le titre abbatial, tout service religieux avait cessé dès 1568 et l'abbaye n'était plus qu'une simple ferme. C'est Noël de Lalane qui y restaura le culte en faisant fermer d'un mur la nef, en partie détruite, créant ainsi une chapelle conventuelle. Il ne parvint cependant pas à recréer une vie monastique : à sa 
mort, ne vivaient à l'abbaye qu'un moine et son valet. Les abbés suivants démembrèrent peu à peu le domaine et l'abbaye redevint ferme ${ }^{40}$.

1665 Constitution $^{41}$. six juillet ${ }^{42}$. /

Grosse. Fut présent en sa personne $\mathrm{M}^{\text {re43 }}$ Noël de La Lane, docteur en théologie / de la maison et societté de Sorbonne, abbé de l'abbaye de Valcroissant, / demeurant à Paris, rue des prouvaires ${ }^{44}$, parroisse St-Eustache en la maison de / Madame Le Bosse, sa sœur, lequel volontairement a recogneu et confessé / avoir vendu, créé, constitué, assis et assigné, vend, crée, constitue, assiet et assigne par / ces présentes de tout, dès maintenant à tousjours, et promect de garantir de tous troubles / et empeschemens générallement quelsconques, fournir et faire valloir tant en principal / cours et continuation d'arreraiges que rachapt, à $\mathrm{M}^{\text {re }}$ Toussainct Desmares, / docteur en théologie, demeurant à Liancourt, estant de présent à Paris, logé / en l'hostel de Laincourt, rue de Seyne, fauxbourg St-Germain, parroisse St-/ Sulpice, à ce présent et acceptant acquereur pour luy, ses héritiers et ayant cause / cinq cent cinquante livres de rente annuelle et que led. sr. constituant / promet et s'oblige doresnavant bailler et payer aud. sr. acquereur, sesd. héritiers / et ayant cause $^{45}$, en sa maison à Paris par chacun an, aux quatre quartiers ${ }^{46} /$ accoustumez, esgallement, dont le premier d'iceux escherra le dernier jour de / septembre prochain et continuer ainsy les autres avoir et prendre speciallement / sur la maison et terres en despendant sciz au Mesnil-le-Roy consistant / en un enclos de treize arpents, douze arpents de prez hors led. enclos et ung / arpent et demy de vigne ; item ${ }^{47}$ sur la sixième partie par indivis des charges / du greffier controlleur et commis portesacq ${ }^{48}$ des productions du conseil ; item sur / seize cent trentequatre livres seize sols de rente constituez à desfunct $\mathrm{M}^{\text {re }}$ Claude / Bourcier, receveur des tailles à Sens le XXI juillet $1636 / 2$ cent 52 livres 7 sols de rente sur lesd. tailles constituez le 12 décembre 1635 aud. sr. / Bourcier ; item sur 561 livres 6 sols de rente sur les gabelles constituez aud. sr. / le vingt-cinquième juillet seize cent trente-cinq ; item sur la somme de sept mil livres / deus par lesd. srs. Maire et eschevins de la ville de Sens par obligation passée / par devant Bonot ${ }^{49}$ et Paisant, notaires à Paris, le treize janvier seize cent trente-sept / au proffit de demoiselle Anne de La Lane et les interests d'icelle somme, le tout / aud. sr. constituant appartenant tant en son propre que d'acquisition et encore / comme donnation de desfunct François de La Lane [Bana ?], son cousin, suivant / les donations qu'il luy en a faictes notamment celle de lad. somme de sept mil / livres de principal et interests passée par-devant Lecas et Lesomellier, notaires, le / XIIII febvrier seize cent soixante [...] et ainsy que led. sr. constituant a dict et déclaré / comme generallement sur tous et chacuns les autres biens meubles et / immeubles quelconques présents et advenir dud. sr. constituant le tout qu'il en / $\mathrm{f}^{\circ} 1 \mathrm{v}^{\circ} / \mathrm{a}$ chargé, affermé, obligé et hipotéqué pour fournir et faire valloir ladicte / rente bonne et bien payable par chacun an à tousjours ausd. quartiers, nonobstant / touttes choses à ce contraire, sans que la generalle obligation desroge à la spéciale / et la specialle à la generalle pour desd. cinq cent cinquante livres de rente jouir et / ceste présente constitution faire moyennant la somme de unze mil livres tournois qu'il / est à raison du denier vingt par led. sr. constituant et a confessé et confesse avoir / receu dud. sr. acquereur qui luy a lad. somme baillée, prestée, fournye, comptée / nombrée et deslivrée par lesd. notaires soubzsignés en louis d'argent, le tout bon etc. dont / quictance dessaisissant [...] à la valleur et concurrance de ladicte rente / voullant, procurant, portant donnant pouvoirs racheptables à toujours / lesd. cinq cent cinquante livres de rente en baillant et payant par le racheptables à une toujours / lesd. cinq cent cinquante livres de rente en baillant et payant par le racheptant à une / seulle fois et payement pareille somme de unze mil livres tournois avec les arreraiges / qui en seront lors deubs et escheuz fray mesme et tous loyaux coutsmemes tous / fray de consignation ${ }^{50}$ et droict de controlle ${ }^{51}$ desd. consignations sy aucuns [...] / payer et desbourser nonobstant tous edits et arrests contraires [...] est / desrogé ${ }^{52}$ et renoncé et a led. Delalane déclaré que lad. somme de unze mil / livres par luy emprunctée est pour convertir et employer au 
remboursement / deubz à Monsr. Demontfelon, maître des comptes, de cinq cent livres de rente qui / sont deubs par la ville et habitans de Sens en laquelle led. sr. Bourcier / est sollidairement obligé par le contract de constitution d'icelle, passé par-devant / Bonot et Paisant, notaires à Paris, le douze janvier seize cent trentesept et de laquelle / led. sieur constituans est tenu (sauf son recours) comme donnation dud. sr. / François De La Lane, excuier, qui est un héritier dud. desfunct sr. Bourcier et / possédant les biens de sa succession, promettant led. sr. constituant faire / led. remboursement et par la quictance qu'il en retirera dud. sr. Demontfelon et / déclare que les deniers proviennent de la présente constitution affin que la susd. [...] / rente qui luy appartiendra au moyen dud. remboursement soit et demeure [...] / obliger et hipotequer par privillege special à la [...] et payement et continuation / des 550 livres de rente presentement constituez en principal et arreraiges et mesmes que les biens / immeubles [...] et obligés à icelle par led. desfunct sr. Bourcier par led. / contract de constitution qui sont encorres en nature en sad. succession demeurent / aussi obliges et hipotéqués à lad. rente présentement constituée et à cest effet / led. sr. constituant promet faire et faire faire par lad. [...] la / fo $3 \mathrm{r}^{\circ} /$ subrogation $^{53}$ constituée au proffit dud. sr. acquereur et dès à present il consent / et accorde lesd. subrogations et de lad. quictance qui contiendra lesd. declarations / et subrogation fournir une expédition avec coppye dud. contract de constitution / aud. sr. acquereur dans trois jours prochains a peyne de tous despens / dommages et interests et d'estre contrainct au rachapt de lad. rente à quoy il est / soubzmis et obligé estant aussy convenu qu'arrivant que led. sr. constituant / [...] le rachapt de lad. rente de 5 cent 50 livres et aussy le payement de lad. somme / de 7 livres cy dessus affermés à luy deus par lad. ville et habitants de Sens / et en ce cas ce ne pourra estre qu'en la presence dud. sr. acquereur, sesd. heritiers / et ayant cause pour ce faire appeler en la personne ou au domicile cy-après / esleu pour recevoir sy bon leur semble le rachapt de lad. rente de cent 52 livres tournois / et arreraiges d'icelle en tout ou partie ou stipuller un remploy desd. deniers / [...] d'icelle rente ce qui sera mentionné par lad. quittance / de remboursement et sans que lesd. subrogation et privilège préjudicie / ausd. obligations generalle et specialle ${ }^{54}$ cy dessus ny au contraire, et pour l'execution / des présentes et deppendant led. sr. constituant a esleu et eslit son domicile / irrevocable en ceste ville en la maison de $\mathrm{M}^{\mathrm{r}}$ Leger [...] / [...] scize en lad. rue des prouvaires [...] et aussy led. / sr. Desmares eslist son domicile en la maison de Galloys, l'un des / notaires soubzsignés, pour y recevoir leurs exploits et avis de somation / et coppie de la stipullation cy-dessus. Promettant et s'obligeant etc. Faict et / passé à Paris en lad. maison dud. Galloys, notaire, le sixième / jour de juillet après midy l'an mil six cent soixante-cinq et / ont signé. /

De Lalane abbé de Valcroissant / Desmares / Symonnet / Galloys / 


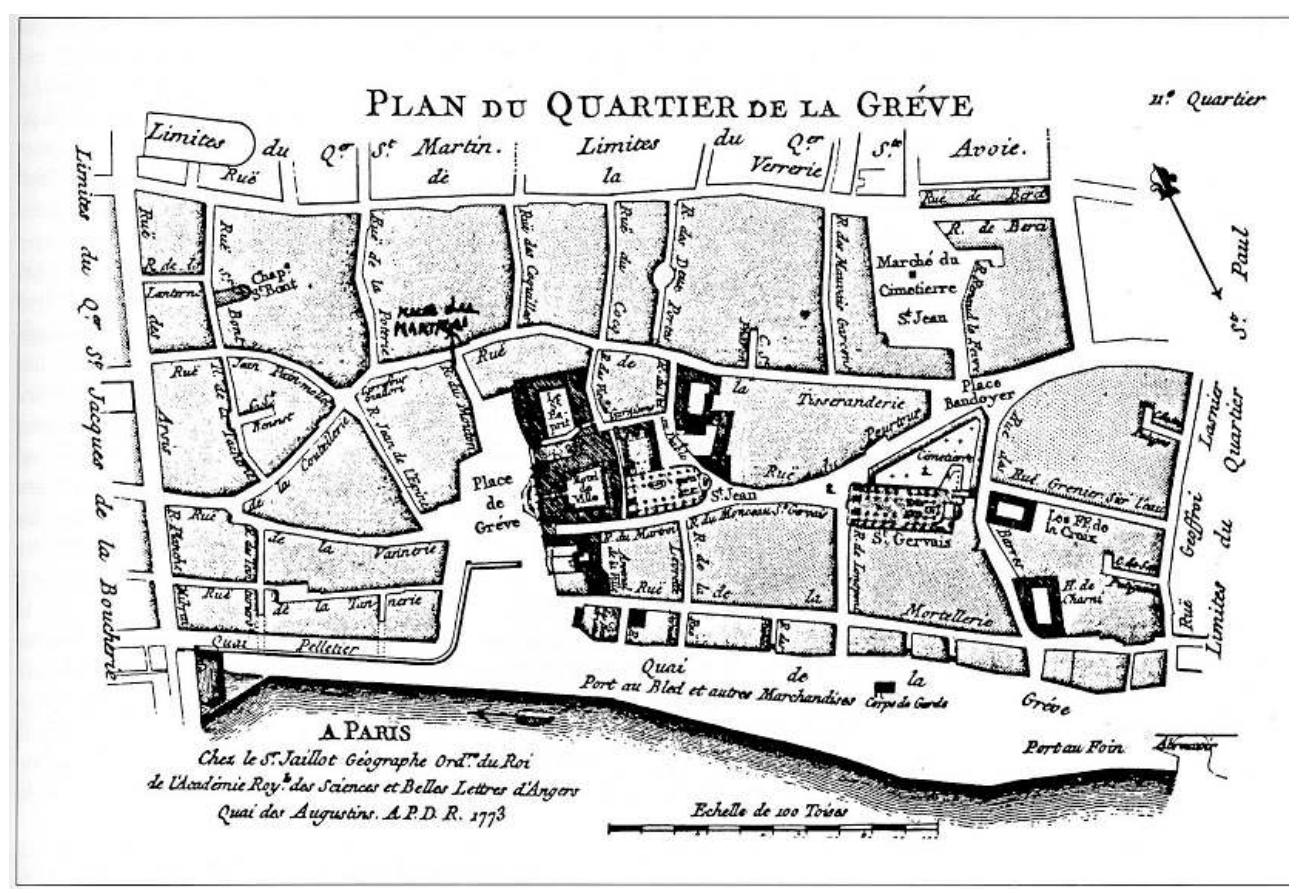

Jaillot, Recherches critiques, historiques et topographiques sur la Ville de Paris (1778-1779), tome III, plan $n^{\circ} 1$ : "Onzième quartier, La Grève. »

\section{Acte $n^{\circ} 4$}

1679 Donnation ${ }^{55}$ Moyen papier vingt-cinq febvrier /

dix-huit deniers la feuille. /

Par-devant les notaires gardenottes du Roy / au chastelet de Paris soubzsignés, fut present en sa personne / Messire Toussainct Desmarre, prestre, docteur en théologie, / demeurant ordinairement au chasteau de Liancour en Beauvaisis / estant de present en ceste ville de Paris, logé a l'hostel de la / Rochefoucaud, sciz rue de Seine, fauxbourg Sainct-Germain, parroisse / Sainct-Suplice, lequel de sa bonne, pure et franche volonté / a fait donnation par ces presentes irrévocable entre vifs et en la / meilleure forme et manière que donnation pour le valloir au sieur / Charles René Desmarre, bourgeois de Vire son nepveu, fils / de deffunt François Desmarre, sieur de Chatillon, frère dud. / sieur donnateur et a damoiselle Jeanne, Françoise et Marie Le Sauvage / aussy niepce dudit sieur donnateur, fille mineure de Pierre / François Le Sauvage, sieur de Bitot et de damoiselle Catherine / Desmarre sa femme, sœur dudit sieur donnateur acceptant / pour led. sieur Bitot leur père et tuteur et encorre / acceptant pour ledit Charles René Desmarre comme son / procureur fondé de la procuration passée par-devant Le / Dannois et Ollivier, notaires royaux à Caen le troisième / novembre mil six cent soixante-seize dont est apparu / demeurée annexée à la presente minutte après qu'elle a esté paraphée / dudit sieur Bitot et à la requisition des notaires soubzsignés / ledit sieur de Bitot, present, demeurant à Caen, en Normandie / estant de present à Paris logé audit hostel de la Rochefoucault / trois cent soixante quinze livres de rente audit sieur donnateur / appartenant et à luy constituez par les vénérables proviseur prime / procureur et Boursier du collège d'Arcourt, fondé en l'université / de Paris, rue de la Harpe, moyennant la somme de neuf mil / livres à raison du denier vingt-quatre, par contract passé / par-devant Carnot et Le Maistre, notaires au chastelet de Paris / le neufvième may seize cent soixante-treize en vertu de l'arrest de / Nos 
seigneurs de parlement du cinqiesme may audit an / transcript aud. contract et pour emploier à l'effect y porté / et huict cens soixante quatorze livres un sol dix deniers de / rente aud. sieur donnateur appartenant à prendre sur l'hostel de / $\mathrm{f}^{\circ} 1 \mathrm{v}$ - / cette ville de Paris au moyen de l'acquisition qu'il en a faite / de dame Margueritte de la Lane, espouze non commune en biens de / Messire François de Belloy, sieur de la Salle et Messire Pierre Marie / de La Lane es noms, par contract passé par-devant de La Balle et Büon / notaires au chastelet de Paris le dixiesme may et autres jours / suivants seize cent soixante-treize estant en quatre parties constituées par / Messieurs le prevost des marchands et eschevins de cette ville de / Paris : la première de cinq cents soixante-une livres cinq sols / à $\mathrm{M}^{\mathrm{r}}$ Claude Boursier le vingt-cinq juillet seize cent trente-cinq sur les / trois millions de gabelles; la seconde de cent quarante-deux / livres sept sols sur le clergé de France comprise audit contract / en une seulle partie et neantmoins estant en deux dont l'une de / quatre-vint-deux livres constituée à Henry Ladvocat le quatre / janvier seize cent soixante-deux, et l'autre de soixante livres sept sols au / sieur Despreau, le septiesme may seize cent soixante-trois; la troisième / desd. quatre parties de quatre-vingt-quinze livres neufs sols dix / deniers de rente faisant partie de dix neuf cent trente-huict livres / quatre sols constituée aud. sieur Ladvocat le vingt decembre mil / cinq cens soixante-sept sur ledit clergé et la quatrieme et dernière / de soixante et quinze livres de rente faisant partie de plus grande / rente constituée à Imbert Bourdon, le cinq may mil cinq cent / soixante-quatre sur ledit clergé pour lesd. rentes de trois cents / soixante-quinze livres d'une part et huict cens soixante-quatorze / livres un sol dix deniers d'arrerages appartenir et estre partagées / esgallement entre lesd. Charles René desmarre pour moityé et / lesd. damoiselles le Sauvage, mineures, pour l'autre moityé tant en / principal qu'arrerages du jour qu'elles commenceront à en jouir / comme il sera dit cy-après, le tout aux charges et reserves cy-/après : scavoir à la reserve que soir ledit sieur donnateur de l'usufruit / desdites rentes données sa vie durant se constituant à cette fin les [...] / à tiltre de precaire desd. donnataires pour estre ledit usufruit après / son decedz reüny à la propriété presentement donnée et à la charge que / sur la moityé appartenant aud. Charles René Desmarre il pourra à / commencer du jour du decedz dudit sieur donnateur cinquante livres. / de rente à damoiselle Desamarre, sa sœur, niepce dudit / $\mathrm{f}^{\circ} 2 \mathrm{r}^{\circ} /$ sieur donnateur, femme du sieur de Vengeon, advocat, à laquelle et aux / sieurs de son côté a légué lesd. cinquante livres de rente demeureront / propres qui seront payables par chacun an soubs faculté de les pouvoir / rachepter en payant à une seulle fois la somme de mil livres à la charge / aussy qu'au moyen de la présente donnation faite ausd. damoiselles Le Sauvage / mineures de la moityé de lad. rente, elles seront tenues de renoncer par un / acte expres aussy tost leurs majorités atteintes à prendre aucunes part / es sucessions paternelle et maternelle desd. sieur et dame Le Sauvage, leurs / père et mère, le total desquelles sucessions demeurera et appartiendra / aux enfants masles lesd. sieur et damoiselle Sauvage sans que / leursd. filles donnataires y puissent rien pretendre sy mieux elles n'aiment / touttesfois partager avec leursd. père et mère lorsqu'elles auront lieux / au terme de la coutume en Normandie, et aussy à condition led. / sieur Le Sauvage survit led. sieur donnateur, il jouira de lad. moityé desd. / rentes donnees à sesd. filles tant qu'elles demeureront sans estre pourvues / par mariage ou en religion et aussy pendant ce temps il sera tenu les / nourrir et entretenir comme il a fait jusqu'à present et ainsy que des / filles comme elles doibvent estre entretenües et sy lesd. filles viennent à / decedder avant leurd. pere sans estre mariée ou sans faire profession en / religion ou ayant estée mariée ne laissant d'enfant vivant $/{ }^{*}\left[{ }^{*}\right.$ ou ayant porté une pension / viagère au monastère où / elles auront fait profession] l'usufruit de la part et portion appartenant à celles des filles qui sera / deceddée dans la moityé de lad. rente presentement donnée appartiendra / aud. sieur Le Sauvage, leur père, sa vie durant seullement, et le fond / et propriété appartiendra / ausd. enfants masles dud. sieur Le Sauvage / sans que les autres filles qui y surviendront y puisseent prétendre en aucune / chose, plus 
seront tenus lesd. donnataires de paier après le décèdz / dud. sieur donnateur à Renee Desmarre, femme du sieur de Les Mondieux / demeurant de present à Rouen et luy faire tenir au lieu où elle sera sans deschet / ny diminution cent cinquante livres par chacun an de pension viagère / dont moityé montant soixante quinze livres par led. Charles-René Desmarre / et lesd. Sauvage, mineures ; l'autre moityé montant à pareils soixante / quinze livres dont lesd. donnataires demeureront deschargez du jour du / decedz de lad. Renée Desmarre, et à la charge aussy que lesd. donnataires / ne pourront pendant la vie dud. sieur donnateur engager, vendre ny / ypotecquer lesd. rentes données. Cette presente donnation ainsy faite / par led. sieur donnateur pour la bonne amitié qu'il porte ausdits / donnataires, ses neveux et niepces et par ce qu'ainsy luy plaist / $f^{\circ} 2 v^{\circ} /$ leur transportant en outre leurs droits de propriété qu'il avoit esd. / rentes s'en dessaisissant à leur proffit, voulant qu'ils en soient saisis / et mis en possession par qui et ainsy qu'il appartiendra constituant / à cette fin son procureur le porteur des présentes au greffe du chastelet de / Paris, lesd. parties ont constitué leur procureur le porteur desd. presentes / luy en donnant pouvoir et d'en requerir acte. Promettant etc., obligeant etc., / renonçant etc. Fait et passé à Paris en l'estude de Galloys l'un desd. / notaires soubzsignes l'an seize cent soixante-dix-sept, le vingt-cinquiesme febvrier / avant midy et ont signé. /

Desmares / Chibert / Le Sauvage / Galloys /

Petit papier / $\mathrm{f}^{\circ} 3 \mathrm{r}^{\circ} /$

Par-devant les notaires royaux à / Caen soubzsignes le mardy trois novembre seize cent / soixante et saize fut present Charles Desmarre / sieur du lieu bourgeois de Vire estant de / present en cette ville, lequel a donné plain / pouvoir, puissance et autorité à Pierre François Le / Sauvage, sieur de Bitot, de pour luy en /* son nom accepte tous et chacuns les advancements / de succession et donations qui pourront estre / faits au proffit dud. sieur constituant par Messire / Toussaint Desmares demeurant en la ville de Paris / et pour cet effet signer et passer tous actes / à ce requit et necessaires soit devant nottaires / ou ailleurs faire les [...] esleu domicile [...] / faire aux fins d'insinuation dyceux mesme / de resepvoir tous deniers de telle somme qu'il / apartiendra en faire les poursuites et en donner / quittance egalement etc., quittant etc., obligeant etc., / present Estienne Crestien et Denis Gilles Le / Picard dud. Caen [et ont signé] /

Charles Desmares / Le Dannois / D. Le Picard / Ollivier / Crestien /

*Paraphé suivant / l'acte passé par-devant / les notaires soubzsignés ce / jourd'huy vingt-cinqième / febvrier seize cent soixante / dix-sept / [signé] Le Sauvage / Galloys / Chibert /

Scellé le 3 novembre / 1676 /

\section{Acte $n^{\circ} 5$}

5 Alors que Jeanne de Schomberg, duchesse de Liancourt s'est éteinte le 14 juin 1674 et que son époux l'a suivie dans la tombe le $1^{\mathrm{er}}$ août de la même année, l'acte suivant, en date de $1683^{56}$, montre que Toussaint Desmares vivait toujours au château de Liancourt. C'est d'ailleurs aux côtés de ses protecteurs, dans la chapelle du château, qu'il sera inhumé57.

1683 Procuration. Vingt-quatre mars. /

Par-devant François Monnet, nottaire royal, / gardenotte heredittaire au gouvernement, bailliage / et comté de Clermont en Beauvaisis ${ }^{58}$, / résidant au bourq de Liancoure / soubzsigné et en présence des témoings cy après / nommez, comparut personnellement vénérable ${ }^{59}$ / et discrette ${ }^{60}$ personnes $\mathrm{M}^{\mathrm{e}}$ Toussainct Desmares / prestre, docteur en Théologie, demeurant au / Chasteau de Liancourt, lequel a faict et constituée / son procureur ${ }^{61}$ general et special $\mathrm{M}^{\mathrm{e}}$ Germain / Chaumoret, bourgeois de Paris et demeurant places / de greve soubz le pillier du sainct esprit près le / grand bureau des pauvres ${ }^{62}$, parroisse Sainct Jean, / auquel il 
a donné pouvoir et jouissance de pour / luy et en son nom recepvoir de Messieurs les / conseillers du roy, tresoriers recepveurs et paieurs / des rentes de l'hostel de ville de Paris et de tout / autre qu'il appartiendra tout et ung chacuns les / arrerages escheus et quy escheront cy après / des rentes aud. sieur appartenants constituee sur / led. hostel de ville de telles sommes qu'elles pourront / estre; et assignées sur le clergé, aydes et gabelles / du receu desdits arrerages se tenir par ledit / procureur pour comptent en donner faire et / passer par eux telles quittances et descharges / que besoings sera et au resceus de payement / desdits arrerages faire contre les debitteurs / substituer en son lieux et places telle personne que / $\mathrm{f}^{\circ} 1 \mathrm{v}^{\circ} /$ ledit procureur advisera bon estre pour faire laditte / recepte à conditions que ledit procureur cy dessus / nommez, demeurera garand des deniers quy seront / receus en tiltre de la presente procuration et a déclaré / ledit sieur constituant avoir esté entièrement payé / et satisfaict des arrerages precedent par ledit / sieur procureur recepveur sçavoir sur le clergé les / arrerages escheue jusque et compris le quartier / d'octobre mil six cent cinquante cinq, promettent et / obligeant etc. renonçant etc. Faict et passé audit / Bourcq de Liancoure, l'an mil six cent quatre-vingt- / deux le dix neuf ième jours de mars avant midy et a / ledit sieur Desmares signé en présence de Jean Courtois / et Charles Bricongné l'ayné, vignerons, demeurant / à Liancoure - tesmoings ad ce apellé pour l'absence / autres nottaires. /

Charle Bricongné / Desmares / Monnet / Jean Courtois /

Scellé les ans et jour / susdits /

Aujourd'huy est comparu pardevant les notaires / gardenottes du roy au Chastellet de Paris soubsignez / led. Germain Chaumoret, procureur nommé par / la procuration de l'autre part escritte, lequel a / apporté à Galloys, l'un desd. notaires, laditte / procuration pour icelle mettre et garder au rang / $\mathrm{f}^{\circ} 2 \mathrm{v}^{\circ} /$ de ses minuttes et luy en dellivrer des expeditions et / coppies quand besoin sera, ce faissant, icelluy Germain / Chaumoret et Guillaume Chaumoret, bourgeois de / Paris, y demeurant place de Grève, parroisse St Jean / ont ensemblement certifié à tous qu'il appartiendra / lad. procuration estre bonne vallable et véritable / dont led. Germain Chaumoret a requis acte à luy / octroyé par lesd. notaires soubzsignez en leur / estudes à Paris l'an seize cent quatre-vingt-deux le / vingt-quatriesme mars et ont signé /

Chaumoret / Chaumoret / Symonnet / Galloys. /

6 L'étude de ces actes notariés ne renouvelle certes pas la connaissance que nous avons maintenant du Père Toussaint Desmares. Ils attestent que, sans doute en raison des poursuites et des persécutions dont il fut l'objet, Toussaint Desmares vivait dans un milieu très fermé, celui des fervents jansénistes comme Noël de Lalane qui fit avec lui le voyage de Rome pour plaider la cause de Port-Royal. Si la condition de leur naissance les séparait peut-être, leur science en matière de théologie, l'ardeur de leurs convictions et leur rigueur morale les unissaient. Enfin, grâce à ces actes, nous pénétrons un peu dans la vie quotidienne, avec ses modestes soucis matériels, d'un homme que l'on ne connaissait que par sa vie spirituelle. Le nombre très restreint d'actes passés par Toussaint Desmares, la modicité des sommes en jeu, prouvent bien que jamais sa vie spirituelle ne fut entachée par l'attachement aux biens temporels. 


\section{NOTES}

1. Mesnard Jean, Documents du Minutier central concernant l'histoire littéraire (1650-1700), Paris, P.U.F., 1960.

2. Batterel Louis (Père), Mémoires pour servir à l'histoire de l'Oratoire, p.p. A.M. Ingold et E. Bonnardet, Paris, Picard et fils, 1902. Sur ce Père, on pourra se reporter aussi à Moreiri, Le Grand dictionnaire historique ou le mélange curieux de l'histoire sacrée et profane, Paris, Libraires associés, 1759 ; Migne (M. l'Abbé) Encyclopédie théologique, Paris, Ateliers catholiques du Petit-Montrouge, 1847 et Dom Rivet de La Grange, Nécrologe de l'abbaïe de Notre-Dame de Port-Royal, ordre de Cîteaux, Amsterdam, Nicolas Potgieter, 1723.

3. Lesaulnier Jean, Port-Royal insolite, édition critique du recueil de choses diverses, Paris, Klincksieck, 1992, p. 721-735, passim et aussi J. Lesaulnier et A. McKenna, Dictionnaire de Port-Royal (à paraître).

4. Philippe Galloys, notaire, étude LXXV du Minutier central. Il fut notaire du 30 juillet 1636 à 1685 (on ne connaît ni le jour ni le mois de la fin de son activité). Son étude était située rue Saincte Avoye.

5. Nécrologe de Port-Royal, p. 486.

6. M.C./ET/LXXV/122.

7. «Les notaires du Chastelet ont maintenant la qualité de conseillers du Roy et Gardenottes » (Fur.). Sur les notaires, voir M.-F. Limon, « La compagnie des notaires au Chatelet de Paris sous le règne de Louis XI », p. 77-86, in Notaires, notariat et société sous l'ancien régime, Actes du colloque de Toulouse, 15 et 16 décembre 1986, Toulouse, Presses universitaires du Mirail, 1990.

8. Clerc signifie aujourd'huy, un jeune homme qui est destiné à l'estat Ecclésiastique, et qui en a pris le premier caractère, qui est la tonsure (Fur.). La quête de renseignements sur Jean Dezaleux et son frère François, qui se porte caution, a été infructueuse.

9. L'église Sainte-Avoye est aujourd'huy disparue. "Sainte Avoie était très populaire à Paris, à cause du transfert de ses reliques à l'abbaye de Saint-Antoine » (Réau Louis, Iconographie de l'art chrétien, Paris, P.U.F., 1958, t. III. p. 165).

10. «Messire : titre ou qualité que prennent les Notaires, \& les personnes de qualité dans les Actes qu'ils passent, au lieu de celle de maistre qu'on donne aux graduez. [...] Messire tel Abbé » (Fur.). Ainsi Gallois nomme « Messire » Toussaint Desmares, mais « maistre », François Dezaleux, avocat.

11. Le prieuré sante-Avoye se trouve rue Sainte-Avoye, c'est-à-dire la portion sud de la rue du Temple, à l'angle nord de la rue Geoffroy l'Angevin. Voir Jaillot (Jean-Baptiste Renou de Chauvigne dit), Recherches critiques, historiques et topographiques sur la ville de Paris avec le plan de chaque quartier, (1778-1779) 5 vol. in-8. Plan du dixième quartier : saint-Martin-des-Champs, t. II, p. 1.

12. Si l'on ne trouve pas de rue du «Marturey», il existe une rue du Martroi ou Marteret ou Martrai ou encore Martroi Saint-Jean dans le voisinage de l'église Saint-Jean-en-Grève. Il doit s'agir de cette rue qui a disparu lors des agrandissements de l'Hôtel de ville (Jaillot, op. cit., t. III, plan p. 1.

13. Procureur. Qui est chargé de la procuration d'autruy, qui traite en son nom (Fur.).

14. À " procuration », Furetière précise qu'il y a des procurations générales, d'autres spéciales. Il faut, ajoute-t-il, des procurations spéciales pour appeller, pour s'inscrire en faux, pour encherir, pour affirmer des voyages, \&c. 
15. Subside : nom général qu'on donne à toutes les impositions qu'on fait sur les peuples, ou sur les marchandises, au nom du Roy, ou de l'Estat, pour subvenir à ses nécessités, à ses charges (Fur.). Par cette clause, T. Desmares s'engage à n'accepter jamais qu'une rente annuelle de 100 livres et, en cas de nouvelle imposition royale ou ecclésiastique, à en déduire le montant de sa rente. Rappelons que le P. Desmares était «fort peu accomodé des biens de la fortune » (Moreiri, op. cit., p. 216).

16. Tournois, est aujourd'huy une designation d'une somme qui est opposée à parisis. Cent livres tournois, c'est cent livres justes en quelques monnoyes que ce soit. [...] Ce mot ne sert plus que pour oster l'equivoque du mot livres, afin qu'on ne prenne pas pour un poids, ce qui n'est qu'une monnoye (Fur.).

17. Fruits, se dit encore de toutes les choses qui commposent le revenu d'une terre, d'un Benefice, non seulement en grains, mais en cens, rentes, dismes, bois moulins, peages \&c. \& autres redevances (Fur.).

18. A la charge : Adverbial. À condition (Fur.).

19. Obliger, se dit aussi en parlant des obligations civiles \& volontaires. On s'oblige à payer, quand on respond pour un autre (Fur.).

20. Dette. On dit Faire sa dette de quelque chose, pour dire, Répondre pour quelqu'un, s'obliger pour luy à faire ou payer ce qu'il a promis, écrit Furetière, qui donne comme exemple « Une dette solidaire ».

21. Reservation. Terme du Palais. Action ou clause par laquelle on retient, on reserve des pensions, ou des Benefices (Fur.).

22. Lettre, est aussi un titre qui donne le droit de jouïssance de quelque chose, ou l'instrument avec lequel on justifie une prétention (Fur.).

23. Paction. Convention, clause qu'on met dans quelque contract, ou traité. On fait serment dans les procurations ad resignandum, qu'il n'y est intervenu aucun dol, fraude, simonie, ou autre paction illicite (Fur.).

24. Grosse : Actes de Justice, ou procedures escrites en grosses lettres, qui est opposée à minutes, \& aux copies qu'on signifie. [...] Grosse et aussi une expedition en parchemin des contracts, des obligations, arrests \& sentences que delivrent les Notaires \& les Greffiers, \& qui sont executoires quand elles sont scellées (Fur.).

25. «... L'advocat au Parlement, qui ne fait que l'aider, écrire, et consulter... » (Fur.).

26. Pour «fidejusseur» Furetière donne: «vieux mot de Palais qui signifioit ce qu'on nomme aujourd'hui caution ».

27. Domicile. Terme de Pratique, qui se dit de la maison où quelqu'un habite, ou de celle qu'il a choisie, où on peut s'adresser pour faire toutes les significations de ce qu'on luy voudra faire sçavoir. [...] L' Ordonnance veut qu'en tous les contracts [...] on fasse une election de domicile, c'est-à-dire qu'on marque un lieu où on se puisse adresser en execution de l'acte.

28. Quittance: acte par lequel on descharge quelqu'un d'un payement, d'une tête, on le descharge de ce qu'il devoit faire, ou acquitter (Fur.).

29. M.C. / ET / LXXV / 127.

30. Claude de Ferriere, La science parfaite des notaires ou le moyen de faire un parfait notaire, Paris, Charles Osmont, 1686, ouvre le chapitre consacré aux quittances par cette définition : « Quittance est un acte qui sert à justifier du payement d'une somme à laquelle on etoit obligé. Quand une quittance est faite en conséquence d'une obligation ont fait endosser la quittance en icelle, qui doit estre rendue à celui qui paye le contenu dans l'obligation » (p. 406).

31. J. Lesaulnier a établi la chronologie de la vie des Liancourt, op.cit., p.697-715. Roger du Plessis, duc de Liancourt (1598 - $1^{\mathrm{er}}$ août 1674) et Jeanne de Schomberg, sa femme, (1600-14 juin 1674). 
32. Arrerages. Cours d'une rente annuelle \& est constituée, ou d'une pension des cens \& droits Seigneuriaux, ou des loyers de terres et de maisons. [...] Les arrerages monteront plus que le principal. L'Interest ne se dit que des obligations (Fur.).

33. Principal : capital d'une somme dûë, \& est opposé à intérests ou despens.

34. Le dernier dix-huit indique le "prix de l'argent qui court à l'intérest » (Fur.). L'intérêt à verser sur la rente promise se montait donc à dix-huitième du principal soit soixante-quatre livres et cinq sols.

35. Content [...] Celuy qui est content se contient en quelque manière, parce qu'il ne souhaitte plus rien (Fur.).

36. Prejudice. En termes du Palais, se dit d'un grief. [...] On dit aussi dans les quittances, Sans prejudice du surplus... (Fur.).

37. M.C. / ET / LXXV /

38. Noël de Lalane (Paris 1618-23 février 1673), fils de Pierre de Lalane, conseiller et secrétaire du roi, et d'Anne d'Alesso par laquelle il se rattachait à la famille de saint François de Paule, était une famille noble originaire de Guyenne. Prêtre, il obtint le 19 mai 1644 le titre de docteur en théologie à la Sorbonne. Le Nécrologe de Port-Royal, p. 100, donne curieusement " prêtre, docteur en théologie de la Maison de Navarre ». Il fut l'un des députés envoyés à Rome pour plaider, avec le Père Desmares, la cause des jansénistes auprès d'Innocent X. Les deux hommes se connaissaient donc très bien. L'abbé Migne, dans l'article qui lui consacre, analyse trente-six des ouvrages dont il fut l'auteur et ajoute qu' « on lui attribue plus de quarante ouvrages différents » et [...] « outre les ouvrages dont Lalane est seul l'auteur, il en est d'autres qu'il fit en commun avec Arnauld, Nicole etc. » (Encyclopédie théologique, p. 594-604). Voir aussi J. Lesaulnier, op. cit., passim).

39. Il signera du nom de Bonlieu un de ses ouvrages. (voir Migne, op.cit., p. 600). Il chargea Jacques Desmaisons de prendre possession de sa charge de Bonlieu le 22 novembre 1641 mais, dès 1644, il visitait ses abbayes de Bonlieu et Valcroissant. Ces deux filles de l'abbaye de Bonnevaux, aujourd'hui disparue, furent fondées au XII ${ }^{\mathrm{e}}$ siècle et Valcroissant, sise au pied du Glandas, dans un cirque isolé où les sources abondent, étaient tombées en commende dès 1451. Les revenus de Valcroissant étaient dérisoires : «Abbatia Vallis Crescentis in montibus que recepit in diocesi Dien. Et pro ipso membro duntaxat...10 fl. » (J. Chevalier, le diocèse de Die en l'année 1644, procès-verbal d'une visite pastorale avec une étude sur le régime bénéficiaire, Valence, Imprimerie et lithographie de Jules Céas \& fils, 1614, p. 234). Qu'il nous soit permis de remercier M. Legaut qui conduisit notre visite de l'abbaye et $M$. Serge Durand qui nous en dévoila la richesse architecturale cachée.

40. L'abbaye fut inscrite en 1932 à l'Inventaire supplémentaire des monuments historiques et son classement, qui a permis d'en sauvegarder les restes, date du 25 octobre 1971. Sur l'abbaye de Valcroissant : Cîteaux dans la Drôme, Revue Drômoise, juin 1980, t. LXXXIII, p. 49-154 ; L'abbaye de Valcroissant, n.s., Association universitaire d'études Drômoises, bulletin $\mathrm{n}^{\circ} 14$, Valence, C.R.D.P., novembre 1967; U. Chevalier, Cartulaire de l'Abbaye N. D. de Bonnevaux au diocèse de Die, coll. Documents historiques inédits sur le Dauphiné, $7^{\mathrm{e}}$ livraison, Grenoble, Imprimerie F. Allier père et fils, 1889 ; L'Abbaye de Valcroissant, Centre de Formation du Diois. Éducation nationale, Die, Éditions A. Die, 1991 ; J. Chevallier, L'Abbaye de Notre-Dame de Valcroissant de l'ordre de Cîteaux, Valence, Imprimerie de Jules Céas et fils, 1898.

41. Les constitutions de rente étaient fréquentes au XVII ${ }^{\mathrm{e}}$ siècle. Noël de Lalane, par cet acte, crée une rente à son ami.

42. M.C. / ET / LXXV / 128.

43. Messire : Titre ou qualité que prennent les Nobles, \& les personnes de qualité dans les actes qu'ils passent au lieu de celle de maistre qu'on donne aux graduez [...] Messire tel Abbé d'un tel lieu. (Fur.).

44. Voir Jaillot, op. cit., t. 2, p. 1, septième quartier, plan du quartier saint-Eustache. «Rue des prouvaires. Elle fait la continuation de la rue du Roule et aboutit à la rue Traînée, en face du 
portail méridional de S. Eustache. Le véritable nom de cette rue est celui des Prévoires ou Provoires, c'est-à-dire, des prêtres, parce que dès le XIII ${ }^{\mathrm{e}}$ siècle les prêtres de $\mathrm{S}$. Eustache y demeuroient. « La rue des prouvaires existe toujours dans le voisinage de l'église mais la configuration des lieux a changé et la rue des prouvaires se trouve entre la rue du Roule et la rue Supervielle, entre le Pont-neuf et la place René Cassin.

45. Cause, en termes de Palais, signifie un droit acquis à quelque personne par quelque titre que ce soit, vente, cession, donation, succession, confiscation, \&. Ainsi on dit, Ses héritiers ou ayants cause. (Fur.).

46. Quartier, on dit [...] quartier d'une rente, d'un terme, d'une pension, pour dire, ce qui est écheu pendant trois mois, ou le quart de l'année (Fur.).

47. Item, adverbe dont on se sert dans les comptes, dans les états que l'on fait, pour signifier de plus, semblablement (Littré).

48. Sac, en termes de Palais, se dit de celuy où l'on met les papiers d'un procès (Fur.). Pour porter les papiers des affaires qu'ils avaient en charge, les notaires, les avocats, attachaient les différents sacs à leur ceinture.

49. Jean Bonot (1663-1707), étude LXVII.

Valoir, signifie aussi, Garentir, répondre qu'une chose est bonne. Les Notaires mettent dans tous leurs contracts, Avec promesse de garentir, fournir, \& faire valoir.

50. Consignation : action par laquelle on depose en main seure une somme de deniers, des billets ou papiers, en attendant la decision de quelque difficulté, ou que la condition soit escheüe, qui empêche de les délivrer [...] Chaque consignation est de quatorze écus \& demy pour chaque heure de vacation (Fur.).

51. Controlle, registre double qu'on tient des expéditions, des actes de Finance \& de Justice, pour en assurer la conservation \& la vérité [...] Controlle est aussi le droit qu'on paye pour ce controlle (Fur.).

52. Déroger : faire un acte ou une disposition contraire à une précédente, qui luy oste sa validité (Fur.).

53. Subrogation : action par laquelle on est mis en la place, aux droits d'un autre (Fur.).

54. Special. Ce qui a une designation particuliere, qui est opposé à general [...] Les Notaires mettent toûjours dans leurs contracts, Sans que l'hypotheque speciale deroge à la generale (Fur.).

55. Donation Contract, acte public par lequel un homme transmet à un autre la propriété ou l'usufruit du tout ou d'une partie de ses biens. Une donation entre vifs, c'est une donation qu'on fait en pleine santé (Fur.). L'acte qui suit est une donation entre vifs.

56. M.C. / ET / LXXV / 217.

57. Sur l'inhumation du père Desmares à Liancourt, voir les précisions que donne Sainte-Beuve, Port-Royal, éd. M. Leroy, Paris, Gallimard, 1955, V, p. 66, note**.

58. Clermont-en-Beauvaisis, maintenant «Clermont » (Oise).

59. Toussaint Desmares avait alors 84 ans.

60. Discret, est aussi une formule de notaires, un titre d'honneur qu'ils donnent aux Curez \& aux Graduez, et principalement, aux Superieurs des Couvents. Venerable \& discrette personne $\mathrm{M}^{\mathrm{r}}$ tel Curé d'un tel lieu, Prieur d'un tel Couvent (Fur.).

61. Procureur. Qui est chargé de la procuration d'autruy, qui traite en son nom. (Fur.).

62. Bureau, se dit aussi des lieux où on traitte les affaires des communautez. Le Bureau de l'Hostel-Dieu, le grand Bureau des pauvres (Fur.). 
INDEX

Mots-clés : Desmares (Toussaint), acte notarié

Keywords : Desmares (Toussaint), notarial deed

\section{AUTEUR}

MARIE-FRANÇOISE BAVEREL-CROISSANT

Institut d'Histoire de la Pensée Classique 